\title{
Quantitative Mass Spectrometry Normalization in Urine Biomarker Analysis in Nephrotic Syndrome
}

\author{
Timothy D. Cummins ${ }^{a, b}$ David W. Powell ${ }^{a, b}$ Daniel W. Wilkey ${ }^{a}$ \\ Makayla P. Brady ${ }^{b}$ Fredrick W. Benz ${ }^{c}$ Michelle T. Barati ${ }^{a}$ Dawn J. Caster ${ }^{a}$ \\ Jon B. Klein ${ }^{a, b, c}$ Michael L. Merchant ${ }^{a, c}$ \\ ${ }^{a}$ Kidney Disease Program and Clinical Proteomics Center, University of Louisville School of Medicine, Louisville, KY, USA; \\ ${ }^{b}$ Department of Biochemistry and Molecular Genetics, University of Louisville School of Medicine, Louisville, KY, USA; \\ 'Department of Pharmacology and Toxicology, University of Louisville School of Medicine, Louisville, KY, USA
}

\section{Keywords}

Proteomics · Biomarker · Tandem mass tag $\cdot$ Nephrotic syndrome $\cdot$ Kidney disease

\begin{abstract}
Chronic kidney disease (CKD) affects 30 million adults, costs $\sim$ \$79 billion dollars (2016) in Medicare expenditures, and is the ninth leading cause of death in the USA. The disease is silent or undiagnosed in almost half of people with severely reduced kidney function. The urine provides an ideal biofluid that is accessible to high-sensitivity mass spectrometrybased proteomic interrogation and is an indicator of renal homeostasis. While the accurate and precise diagnosis and better disease management of CKD can be aided using urine biomarkers, their discovery in excessive protein or nephrotic urine samples can present challenges. In this work, we present a mass spectrometry-based method utilizing multiplex tandem mass tag (TMT) quantification and improved protein quantification using reporter ion normalization to urinary creatinine to analyze urinary proteins from patients with a form of nephrotic syndrome (focal segmental glomerulo-
\end{abstract}

sclerosis [FSGS]). A comparative analysis was performed for urine from patients in remission (RM) versus active disease (DX) flare. Two-dimensional LC-MS/MS TMT quantitative analysis identified over 1,058 urine proteins and 580 proteins with 2 peptides or greater and quantifiable. Normalization of TMT abundance values to creatinine per $\mathrm{ml}$ of urine concentrated reduced variability in 2D-TMT-LC-MS/MS experiments. Univariate and multivariate analyses showed that 27 proteins were significantly increased in a proteinuric DX flare. Hierarchical heatmap clustering showed that SERPINA1 and ORM 1 were increased $>1.5$-fold in active DX versus RM urine samples. ELISA validation of SERPINA1 and ORM1 abundance agreed with our quantitative TMT proteomics analysis. These findings provide support for the utility of this method for identification of novel diagnostic markers of CKD and identify SERPINA 1 and ORM 1 as promising candidate diagnostic markers for FSGS.

(c) 2022 The Author(s)

Published by S. Karger AG, Basel

Jon B. Klein and Michael L. Merchant are co-senior authors. Timothy D. Cummins and David W. Powell are co-first authors.
C 2022 The Author(s).

Published by S. Karger AG, Basel

This is an Open Access article licensed under the Creative Commons Attribution-NonCommercial-4.0 International License (CC BY-NC) (http://www.karger.com/Services/OpenAccessLicense), applicable to the online version of the article only. Usage and distribution for commercial purposes requires written permission.
Correspondence to:

Timothy D. Cummins, timothy.cummins@louisville.edu 


\section{Introduction}

For this report, we analyzed urine samples from patients with focal segmental glomerulosclerosis (FSGS) as proof of principle of development of an innovative method for identifying novel diagnostic markers for chronic kidney disease (CKD). There are two general classifications of CKD, nephritic and nephrotic syndrome [1]. Nephritic syndrome (i.e., IgA nephropathy and lupus nephritis) is characterized by inflammation of the glomeruli and $<3.0 \mathrm{~g}$ /day proteinuria. Nephrotic syndrome (i.e., diabetic nephropathy, membranous nephropathy, and FSGS) develops as damage to glomeruli, resulting in massive proteinuria ( $>3.0 \mathrm{~g} /$ day) and edema. Progression of all forms of CKD is monitored by changes in proteinuria, serum creatinine, and estimation of the glomerular filtration rate. The gold standard for differential diagnosis is percutaneous kidney tissue biopsies and histological characterization of cellular and extracellular proteins in the renal tubules and glomeruli $[2,3]$. Often, even with histopathology and other clinical data, a clear diagnosis is not achievable, and the clinician is left to attempt treatment with protocols developed for populations and not individuals. In light of this, implementation of novel urine protein biomarkers could help to improve our understanding of renal pathology and increase diagnostic accuracy.

Urinary biomarkers have been useful toward prediction of renal function decline in CKD. Peptide markers consisting of 273 urinary peptide fragments (CKD273) from CKD patients were more predictive of renal function decline than albumin $[4,5]$. Extracellular matrix proteins, including remodeling proteins (MMP-2 and MMP-9), have been detected in the urine of FSGS patients in association with corticosteroid resistance in nephrotic syndrome of pediatric populations [6]. Changes in collagen deposition and degradation are indicative of defects in extracellular matrix remodeling in renal diseases and would be useful in determining the progression of the disease [7, 8]. These findings suggest other markers or composite markers of progressive kidney disease could be valuable and relevant to detection of specific renal diseases and aid in accurate diagnosis and efficacious treatment regimens.

Unbiased identification and quantitation of biomarkers can be accomplished by utilizing proteomics and mass spectrometry-based approaches. These methods are global and sample directly from biofluids with minimal processing. Proteins are readily extracted from urine using filteraided sample preparation (FASP) where buffer is exchanged and protein is concentrated then reduced and alkylated followed by protease digestion into peptide fragments that are fractionated by chromatography and identified by mass spectrometry $[9,10]$. High-sensitivity mass spectrometry provides deep proteome coverage and the option to employ quantitative isobaric tagging methodologies. The large dynamic range of protein concentrations in the nephrotic syndrome urine requires either depletion techniques to remove overrepresented proteins or isobaric tagging approaches combined with multidimensional chromatographic resolution of peptides. Depletion approaches have been shown to have little effect on overall identification of proteins when combined with sufficient peptide chromatographic (LC) fractionation preceding MS analysis [11, 12]. Isobaric tagging methods allow for more comprehensive analysis of the urine proteome and avoid potential co-depletion of important low-abundance proteins. Analyzing the unaltered proteome improves the chances of determining the contribution of low-abundance markers of the disease and dramatically improves sample processing speed or the overall throughput rate. High-abundance protein depletion in urine has been applied and appears to show little to no improvement in overall identification of proteins regardless of proteinuria [11-13]. Effects of depletion actually appears to indicate reduced identification rates, especially in proteinuric samples, suggesting co-depletion under high protein concentration circumstances that may actually have a deleterious effect on overall identification $[13,14]$. This is likely due to co-depletion of peptides and proteins that bind to high-abundance blood carrier proteins, such as albumin.

Biomarker identification and quantitation can be achieved using a label-free MS/MS or isobaric tagging methodology. Tandem mass tagging (TMT) methods improve throughput by multiplexed simultaneous sample analysis and reduces variability toward a quantitative platform for comparative analysis when compared to label-free $[15,16]$. Isobaric reporter tags are cleaved during collision-induced dissociation and detected as unique reporter MS/MS spectra derived from the parent ion [1619]. The combined efficiency and multiplexing capacity makes TMT reporters ideal for applications in urine biomarker discovery. Here, we present an innovative TMTbased mass spectrometry approach for discovery of novel diagnostic markers for CKD.

\section{Methods and Results}

\section{Quantitative Proteomic Approach}

Figure 1 illustrates the proteomics workflow analysis of urine from nephrotic syndrome (FSGS) patients during remission (RM) $(n=3)$ and disease (DX) flares $(n=3)$. Urine was collected from patients during RM following therapeutic intervention with steroids and when urine protein concentrations fell to normal levels or during the DX state when urine protein concentration was 
Patient Urine Collection

1. Proteinuria

2. Normal-protein
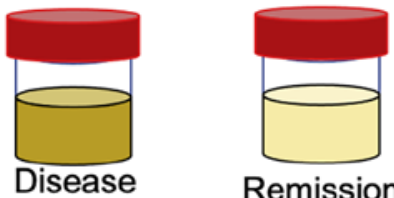

Remission

(Dx)

(Rm)

\section{Concentrate}

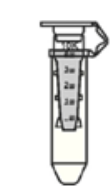

Reduce

alkylate

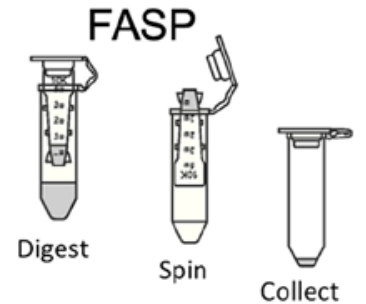

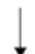

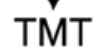

(Isobaric tag multiplex)

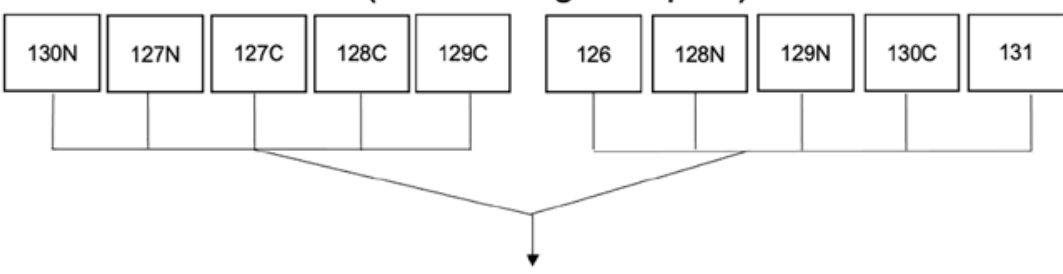

High pH-HPLC

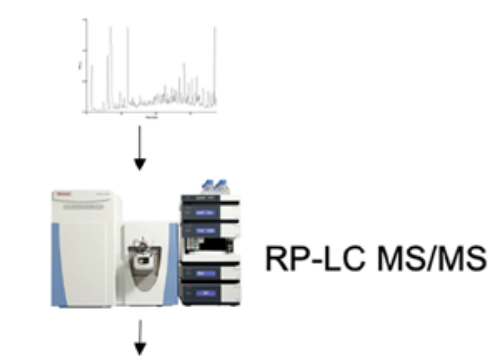

Quantitative Analysis

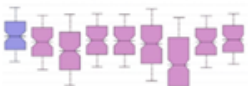

Fig. 1. Quantitative workflow of nephrotic syndrome DX and RM state urine for biomarker identification. 


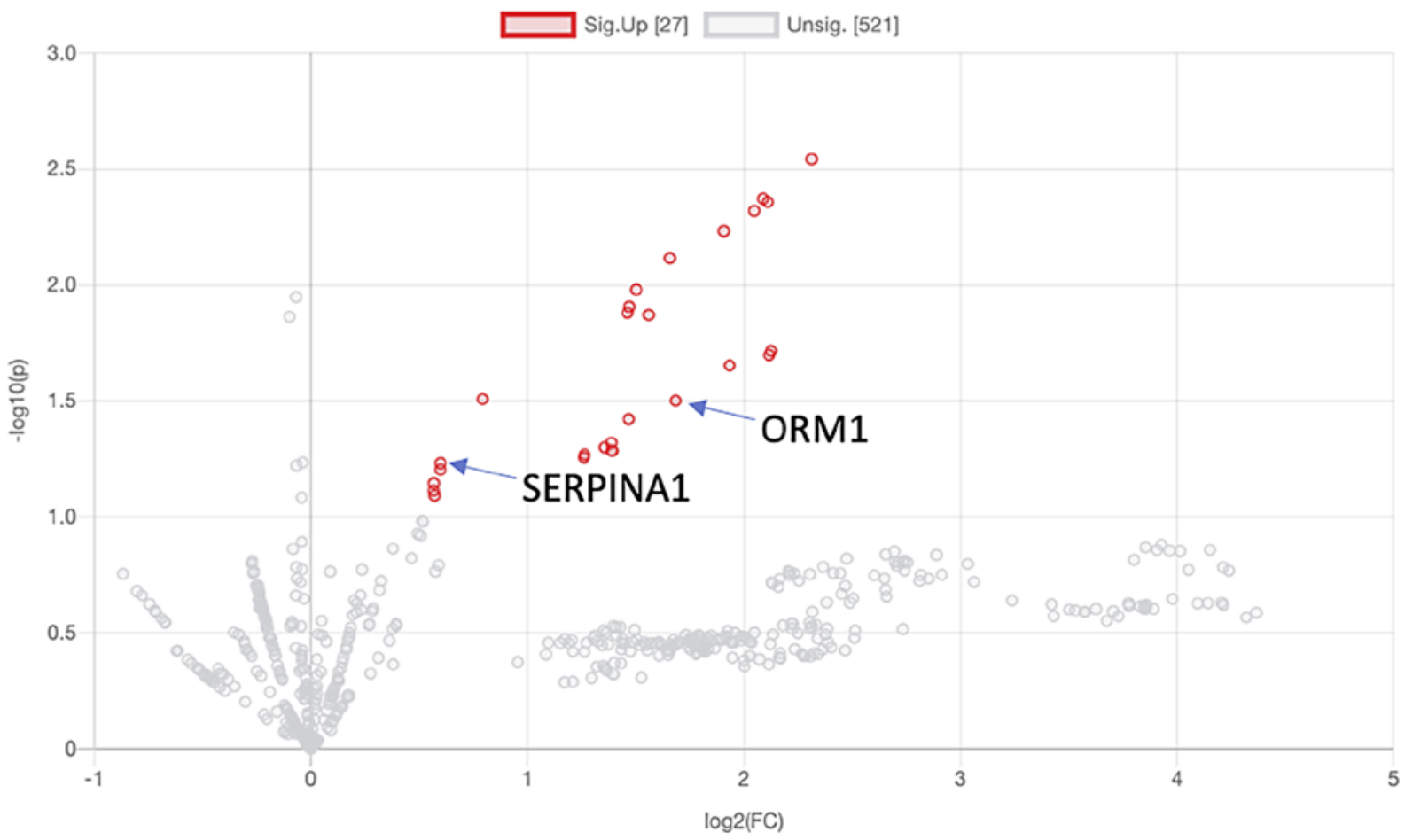

b

\begin{tabular}{rlllll}
\hline & Compounds & FC & log2(FC) & raw.pval & $-\log 10(\mathrm{p})$ \\
\hline 1 & REG1A & 4.9673 & 2.3125 & 0.002866 & 2.5427 \\
2 & SLURP1 & 4.2537 & 2.0887 & 0.0042441 & 2.3722 \\
3 & CANT1 & 4.317 & 2.11 & 0.0043829 & 2.3582 \\
4 & ACAN & 4.1354 & 2.048 & 0.0047853 & 2.3201 \\
5 & SPINK5 & 3.7501 & 1.9069 & 0.00586 & 2.2321 \\
6 & INAFM2 & 3.1553 & 1.6578 & 0.0076539 & 2.1161 \\
7 & FBN1 & 2.8342 & 1.5029 & 0.010465 & 1.9803 \\
8 & TNC & 2.7722 & 1.4711 & 0.012416 & 1.906 \\
9 & CD40 & 2.7551 & 1.4621 & 0.01317 & 1.8804 \\
10 & TRIM33 & 2.9498 & 1.5606 & 0.013451 & 1.8712 \\
11 & LY6H & 4.3628 & 2.1253 & 0.019222 & 1.7162 \\
12 & GLRX & 4.3356 & 2.1162 & 0.020026 & 1.6984 \\
13 & PAM & 3.8208 & 1.9339 & 0.022232 & 1.653 \\
14 & ORM1 & 1.7327 & 0.79306 & 0.031006 & 1.5086 \\
15 & GPR180 & 3.2155 & 1.685 & 0.031511 & 1.5015 \\
16 & SUSD2 & 2.7673 & 1.4685 & 0.037886 & 1.4215 \\
17 & PEBP4 & 2.6183 & 1.3886 & 0.047934 & 1.3194 \\
18 & SPP1 & 2.5608 & 1.3566 & 0.050088 & 1.3003 \\
19 & HEG1 & 2.6217 & 1.3905 & 0.051682 & 1.2867 \\
20 & DNER & 2.625 & 1.3923 & 0.051875 & 1.285 \\
21 & AMPH & 2.4019 & 1.2642 & 0.053863 & 1.2687 \\
22 & FZD8 & 2.3975 & 1.2615 & 0.055446 & 1.2561 \\
23 & SERPINA1 & 1.5147 & 0.59905 & 0.058702 & 1.2313 \\
24 & CCN5 & 1.5143 & 0.59867 & 0.062451 & 1.2045 \\
25 & SERPINF2 & 1.483 & 0.56853 & 0.071579 & 1.1452 \\
26 & HLA-A & 1.4821 & 0.56761 & 0.077033 & 1.1133 \\
27 & IL1RAP & 1.4865 & 0.57191 & 0.080875 & 1.0922 \\
\hline & & & & &
\end{tabular}

Fig. 2. Volcano plot of FSGS nephrotic DX versus RM for TMT fold differences against $-\log 10$ ( $p$ value) urine proteins. a Normalized TMT abundance was submitted to MetaboAnalyst 5.0 to visualize differences in FSGS DX versus RM urine proteomes. b List of 27 significantly increased urine proteins in FSGS DX patient samples. Included in the table are the protein name, FC, $\log 2 \mathrm{FC}$, raw- $p$ value, and the $-\log 10$ ( $p$ value) used to generate the volcano plot. Blue arrows indicate two proteins selected for biochemical validation. FC, fold change. 
a

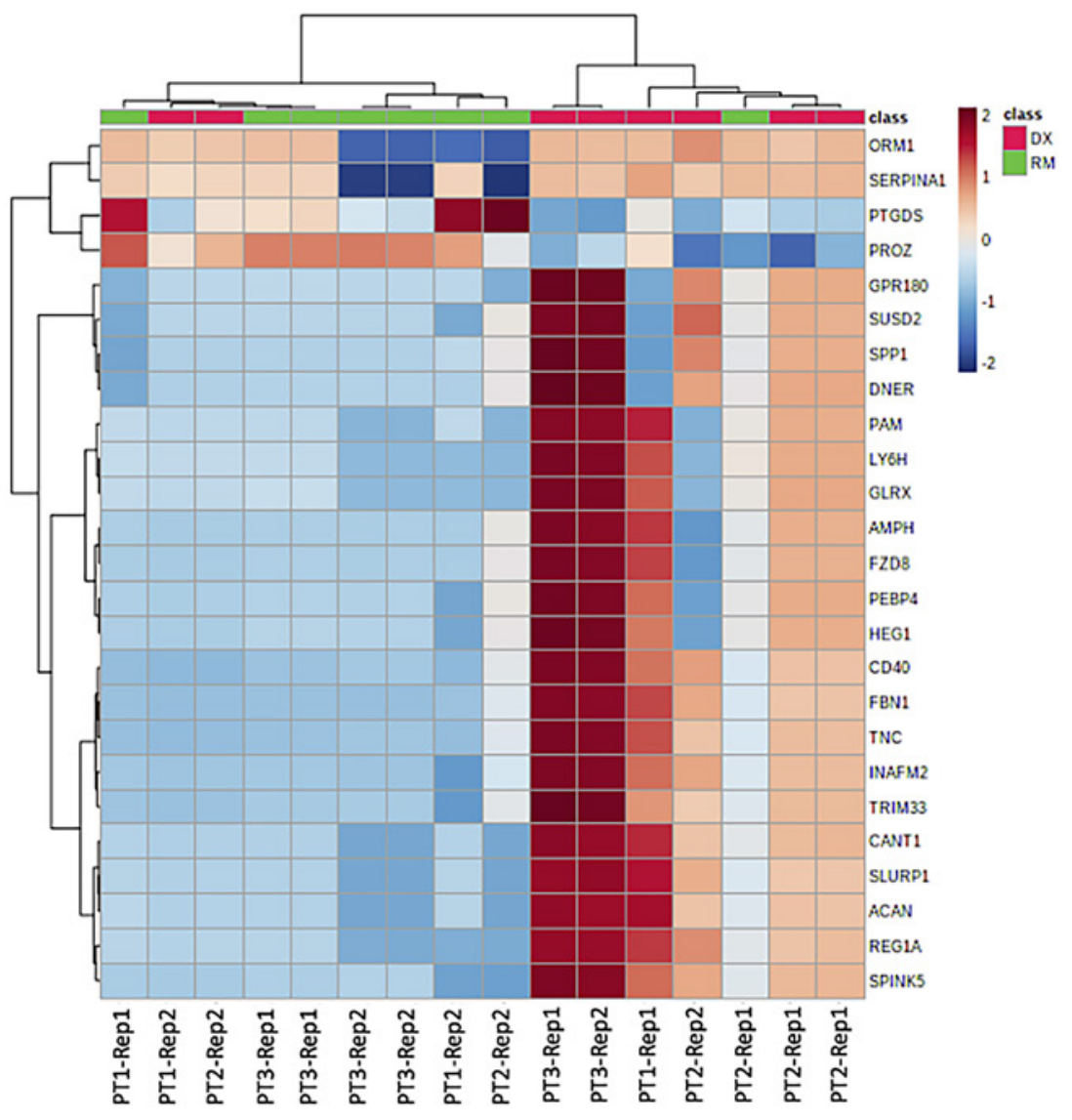

b

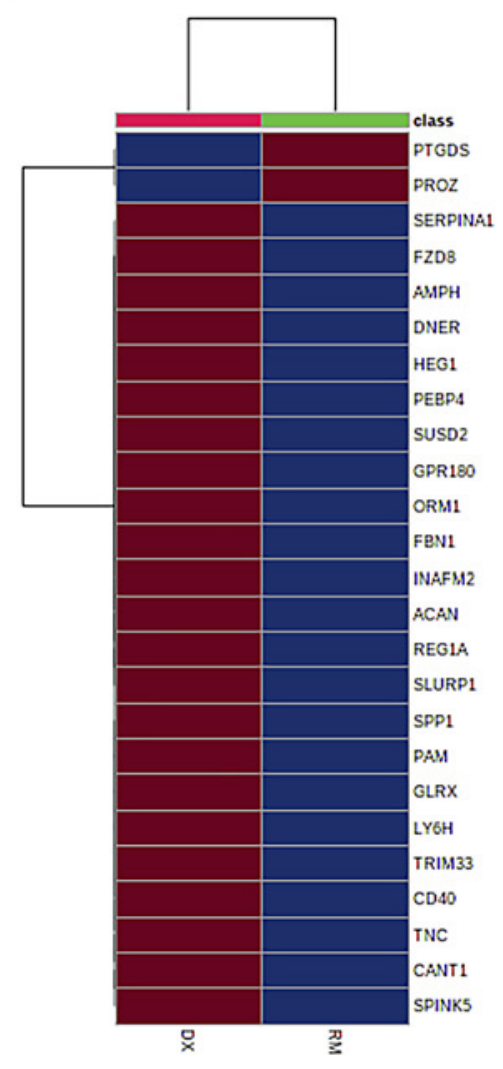

Fig. 3. Hierarchical clustering heatmap of top 25 most significantly altered protein abundance values for replicate TMT-FSGS MS/MS quantitative analysis in the DX versus. RM urine proteome. a Individual replicate analyses expressed as relative abundance across each individual measurement. b Average TMT abundance signal for grouped RM and DX for the top 25 differentially abundant protein values.

above $1,000 \mathrm{mg} / \mathrm{dL}$ (see online suppl. Table 1 for patient clinical information; for all online suppl. material, see www.karger.com/ doi/10.1159/000522217). Urine was immediately stored at $-80 \mathrm{C}$ until analysis was performed. Urine was thawed and protease/ phosphatase inhibitor (HALT cocktail) was added at 1:100 (vol/ $\mathrm{vol})$. Urine was concentrated, and then $50 \mu \mathrm{g}$ of protein was bounded to FASP filters for buffer exchange, reduction, alkylation, and trypsin digestion. Following digestion, $25 \mu \mathrm{g}$ of resultant peptides was labeled with TMT ion reporter tags and further fractionated by high $\mathrm{pH}$ RP-LC. Urine samples were labeled with different TMT tags. Concatenated TMT fractions were then separated by 1-dimensional RP-LC-MS/MS into a high-resolution Q-Exactive mass spectrometer for spectrum acquisition.

\section{Mass Spectrometry Results}

Spectra were submitted to MaxQuant (v1.6.17) to search and filter spectra using strict FDR criteria (1\% for protein decoy and $0.1 \%$ peptide matching to decoy) against a UniProt Human Review
210210 database. This identified 580 proteins with 2 peptides or more assigned and 1,058 total proteins at the 1-peptide threshold. Subsequently, we implemented the Scaffold 5.0 Q + S software package for comparative analysis of each DX and the RM urine proteome sample. MetaboAnalyst 5.0 was used to filter; impute missing values using the $1 / 5$ minimum values rule; generate heatmaps, volcano plots, and orthogonal-orthogonal projections to latent structures discriminant analysis (oPLS-DA) graphs; and conduct basic statistical analysis of the sample groups.

Figure 2 shows protein levels in patient DX and RM urine presented in a volcano plot, where the $x$ axis is log2-FC (fold change), and the $y$ axis is $-\log 10$ ( $p$ value). This analysis yielded 27 significantly increased proteins in DX urine samples at $>1.5$-fold. Figure 3 shows hierarchical heatmap analysis in MetaboAnalyst 5.0 for each replicate analysis limited to the top 25 most significant changing protein abundance values in (a). This analysis allows a granular view of each replicate and the stratification of the patient by RM or the DX state. (b) Average values from (a). Figure 4 shows multivariate analy- 
a

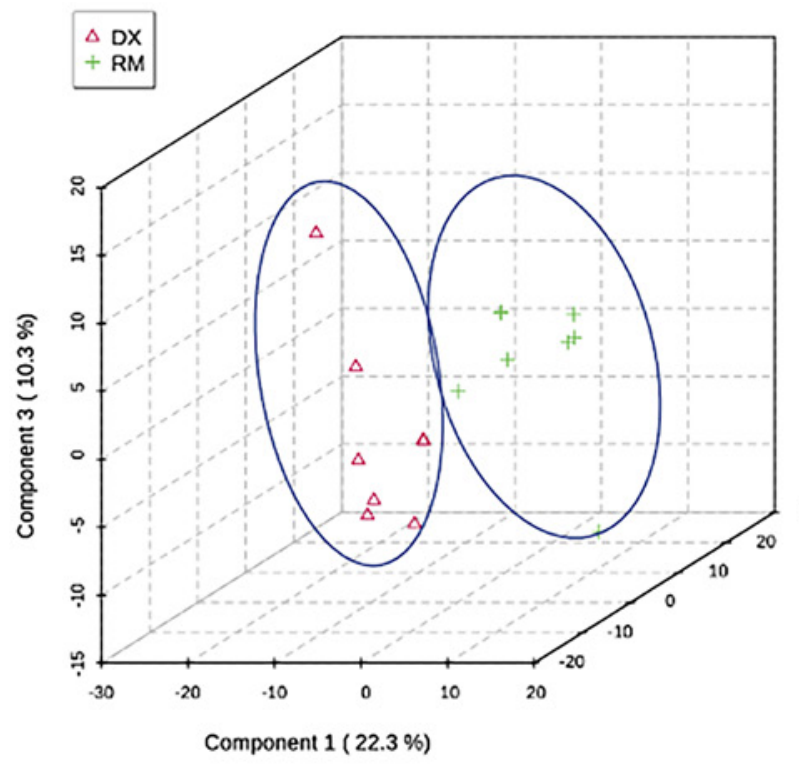

c

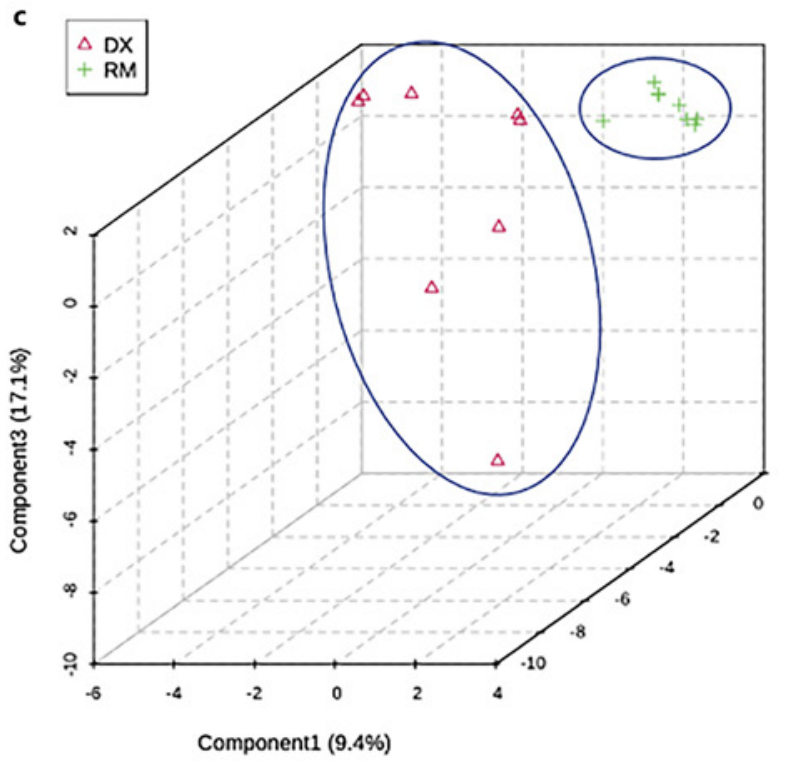

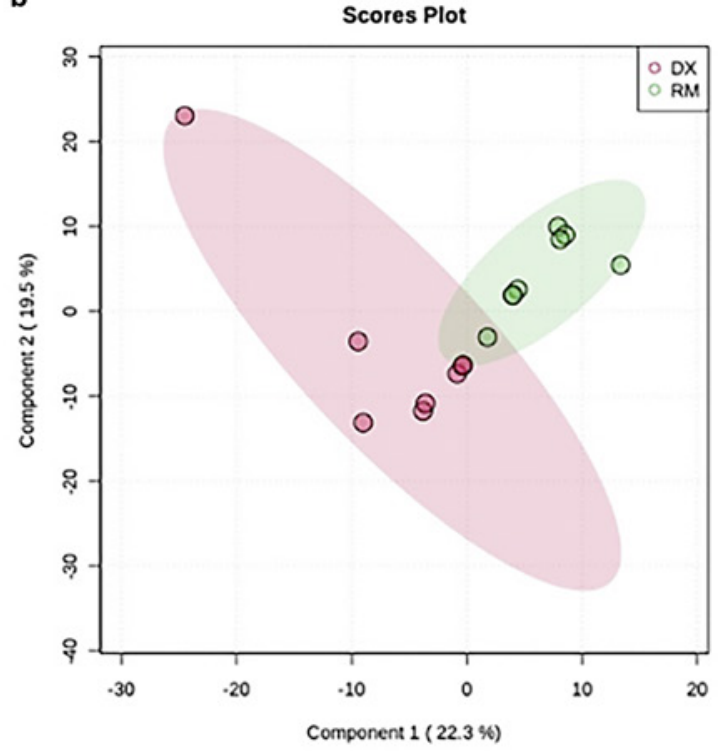

d

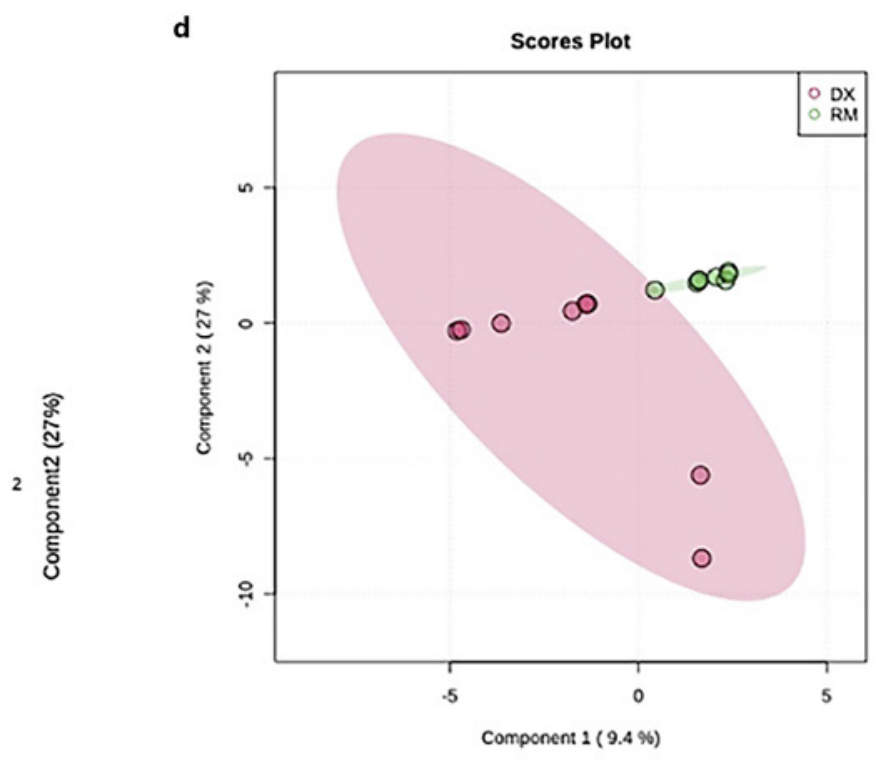

Fig. 4. Multivariate analysis of quantitative urine proteome differences in FSGS DX versus RM patient samples for replicate MS/MS analyses. a PLS-DA (partial-least squared discriminant analysis of DX vs. RM urine proteome components contribution in a 3-dimensional output. b PLS-DA with 95\% CI in 2-dimensional plots with DX (pink) and RM (green). c sPLS-DA 3-dimensional scores plot. d sPLS-DA in 2-dimensional scoring plot with 95\% CI in pink (DX) and green (RM). CI, confidence interval; sPLS-DA, sparse partial-least squared discriminant analysis.

Fig. 5. ELISA validation of TMT mass spectrometry quantitation of urine proteins ORM1 and SERPINA1. Each patient-paired DX or RM sample was measured 3 times independently by ELISA (ng/mL). a, c are unnormalized, $\mathbf{b}, \mathbf{d}$ are normalized to $\mathrm{uCr} \mathrm{mg} / \mathrm{mL}$ per $\mathrm{ml}$ of urine. ANOVA was used to determine statistical significance with a standard $p$ value $<0.05$ accepted as the cutoff.

(For figure see next page.) 


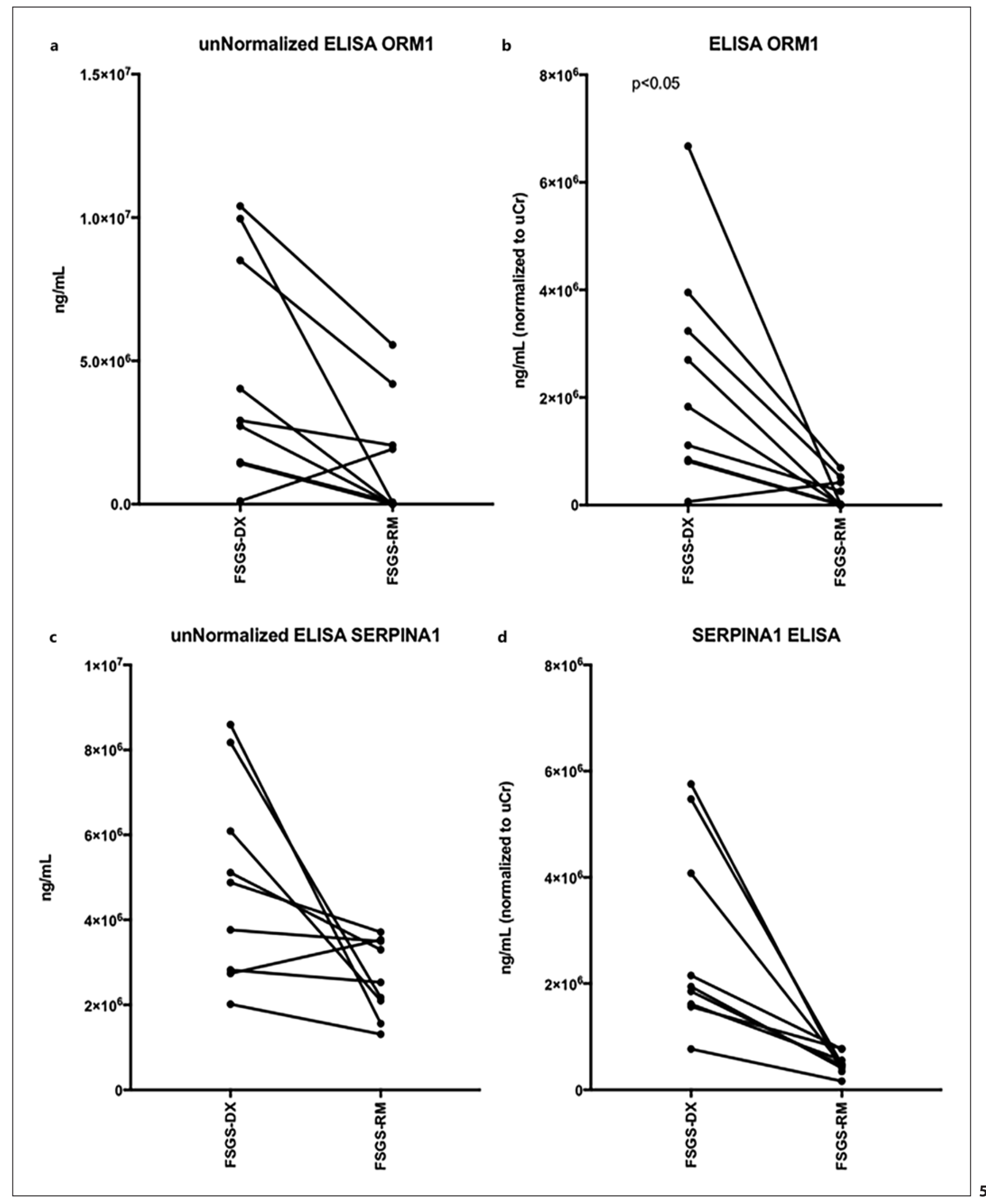

Biomarker Analysis in Nephrotic 
Fig. 6. ORM1 and SERPINA1 quantitation by TMT-MS/MS with and without normalization. a ORM1 levels measured by TMT$\mathrm{MS} / \mathrm{MS}$ and normalized to $\mathrm{uCr}$ per/mL of urine concentrated. b SERPINA1 abundance by TMT-MS/MS with uCr normalization.
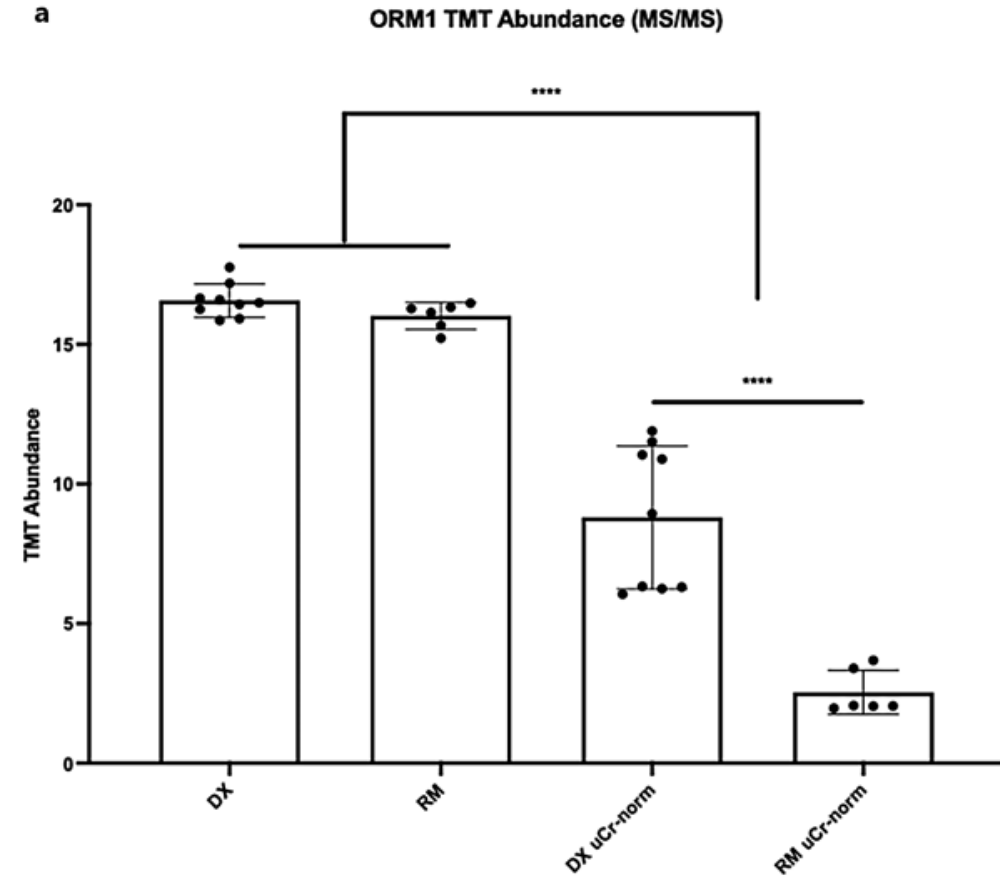

b SERPINA1 TMT Abundance (MS/MS) ....*

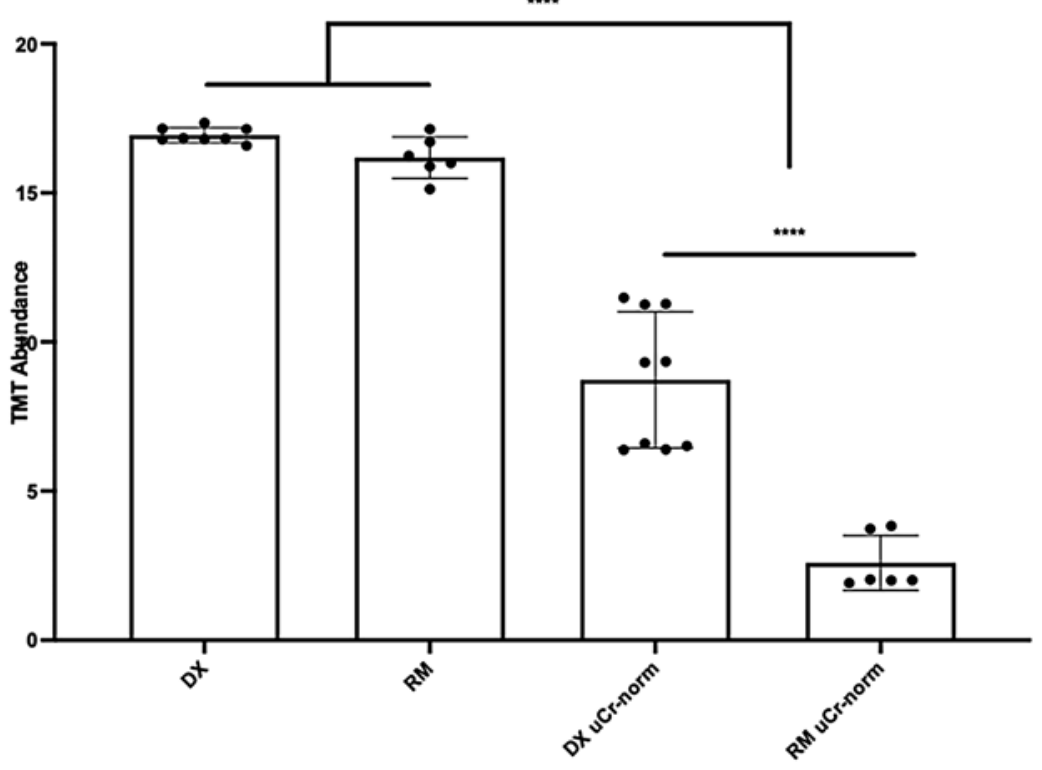

sis of the urine proteome quantitative abundance differences in FSGS DX and RM patients in this study. PLS-DA analysis results in Figure 5a show scoring plots indicating robust stratification of patients based on the quantitative profile. Panel B shows the 95\% confidence interval for each patient category (pink is DX and green is RM). Panel C and D show a similar analysis using sparse PLS-DA. Each method appears to robustly segregate urine proteome abundance esti- mates for DX and RM patient categories, even in a small exploratory dataset. This suggests even a large heterogeneous patient sample set could benefit from this type of analysis.

\section{Data Confirmation/Validation}

SERPINA1 and ORM1 were selected from results for data interpretation and validation experiments. ORM1 (A1AG) and SERPI- 


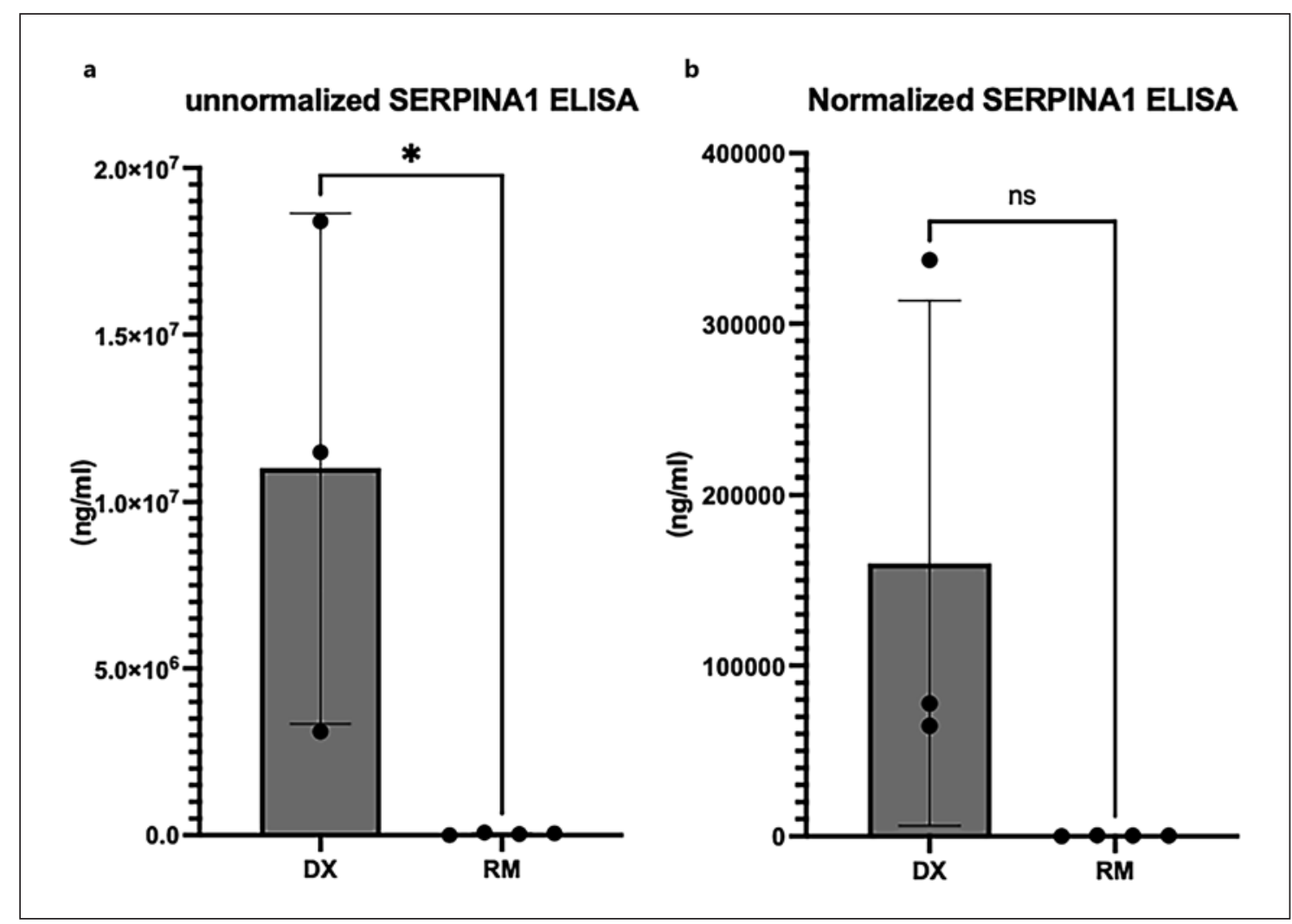

Fig. 7. ELISA validation of SERPINA1 levels in an independent sample cohort. Each patient DX or RM sample was measured 2 times independently by ELISA, and the average value was plotted as $\mathrm{ng} / \mathrm{mL}$. a shows unnormalized SERPINA1 levels, b values are normalized to $\mathrm{uCr} \mathrm{mg} / \mathrm{mL}$ per $\mathrm{mL}$ of urine. Student's $t$ test was used to determine statistical significance with a standard $p$ value $<0.05$ accepted as the cutoff.

NA1 (A1AT) were modestly increased at $\sim 1.7$ - and $\sim 1.5$-fold (Fig. 6b). Figure 6 illustrates normalization of TMT abundance values with urine creatinine $(\mathrm{uCr})$ and the data variation in individual TMT protein quantifications for ORM1 and SERPINA1. These mass spectrometry quantitation results were validated with an ELISA assay for SERPINA1 and ORM1 (Fig. 5) on each patient DX) and RM sample in 3 technical replicates on different days. Abundance levels are expressed as $\mathrm{ng} / \mathrm{mL}$ and normalized to $\mathrm{uCr} / \mathrm{mL}$. Notably, ORM1 and SERPINA1 ELISA normalization with $\mathrm{uCr} / \mathrm{mL}$ markedly improved variability in RM patient urine samples. These findings for SERPINA1 were further confirmed in an independent FSGS cohort (3 DX and 4 RM patients) Figure 7. ELISA results for SERPINA1 were significant $(p<0.03)$, while normalization to $\mathrm{uCr}$ led to more variability and an insignificant $t$ test $(p>0.08)$.

\section{Discussion}

Ultimately, we set out to identify and quantify an array of urinary proteins from nephrotic DX and RM patients with minimal sample processing and simple, effective post-acquisition data normalization. Using urine from DX and RM pa- tients, we applied the FASP protocol and TMT labeling with high $\mathrm{pH}$ chromatographic fractionation to increase coverage of the urine proteome. Normalization of TMT reporter ion intensities to urinary creatinine per $\mathrm{ml}$ concentrated urine. Quantitation of known urinary biomarkers of CKD from a small cohort indicates technical feasibility and usefulness of applying this workflow in larger sample sets to interrogate the urine biomarker proteome in depth.

The major emphasis of this study was to establish a quantitative biomarker workflow protocol to be applied in larger cohorts of 25-100 patient samples. The small sample size used in our method development was not intended to reach statistical thresholds beyond the capability to detect the presence of peptides and proteins and allow for preliminary analysis. Application of this workflow in larger patient sample cohorts will allow for robust analysis and indication of potential biomarkers for more in-depth studies and validation. The workflow presented is straightforward and can be applied in a large-scale fashion to reduce sample preparation variability (technical 
variation) and improve bulk processing of many samples simultaneously. Throughput can be further improved by applying a 96-well FASP approach which reduces cross sample technical variability for larger cohorts [20].

TMT quantitation has not been routinely applied in many human urine biomarker studies to date. One study that successfully implemented TMT quantitative approaches to identify urinary markers of ureteropelvic junction obstruction did identify and quantify some potential pathways involved in disease pathology [21]. This study included robust internal controls for collection of urine from same patient unobstructed and obstructed kidney and identified over 1,100 urinary proteins with 76 being differentially abundant. These proteins were involved in oxidative stress and inflammation pathways. A followup study employed proteomics methods to identify a protein signature or panel of urine biomarkers indicative of ureteropelvic junction obstruction pathology [22]. Studies of this nature indicate the usefulness in applying quantitative proteomics methods in human urine to garner information about disease processes and begin cataloging potential biomarkers and implicating pathways of further interest. Implementation of rigorous quantitation approaches will improve the chances of finding pertinent biomarkers in urine of patients with CKDs and other diseases with effects on kidney homeostasis.

Additionally, complex protein sample analysis requires robust isolation and fractionation techniques to properly delve into the proteome using mass spectrometry methods [23]. In some cases, such as with high-abundance proteins like albumin or uromodulin, 1-dimensional liquid chromatography is sufficient to identify and quantify these proteins but could miss mid-low-abundance proteins. In cases where low-abundance proteins are the main interest, more separation will be required to resolve highabundance protein species from the lesser abundant.

Another consideration is the potential for exosomalor decoy-cell contamination, and our data could contain proteins from decoy cells or other membrane-bound components shed into urine and must be considered in the final candidate biomarker selection process. Another caveat to consider, in comparison to label-free analysis, is that TMT affords multiplexing analysis with the improved reproducibility and throughput. Label-free spectral counting can dig deeper into the proteome and increase single-peptide identifications but leads to higher variability and diminished reproducibility at the lower abundance range of the proteome.

Our current methodological study includes urine from 3 patients with nephrotic syndrome and posttreatment fol- low-up upon RM. The sample set, though small, is ideal for looking at effects of normalization on intra and inter-patient protein quantification. Normalization of quantitative mass spectrometry data often utilizes features within the spectra or the overall dataset (spectral counts, isotopic internal standards, TIC, etc.). Here, we employ clinical information, specifically urinary creatinine from total volume of concentrated urine for each sample. Different volumes of urine were concentrated from low protein $\mathrm{RM}(6 \mathrm{~mL})$ and proteinuric urine $(1 \mathrm{~mL})$ to diminish potential effects of protein loss due to adsorption to the concentrating membrane. It is clear that normalization and multidimensional fractionation can improve the ability to identify low- and medium-abundance proteins in urine within larger sample cohorts. In 2-dimensional analysis, we note that high-abundance proteins (albumin, A1AT, etc.) are accurately quantified following normalization to creatinine but that lower abundance proteins such as ORM1 can be more precisely quantified in DX urine. Utilizing a clinical parameter such as creatinine as a normalization factor integrates the effect of urine concentration or dilution into the protein abundance estimate.

Finally, it is important to consider the goals of a biomarker experiment and chose appropriate methodology to achieve those goals. High-abundance proteins found in urine can be indicators of disease but lack specificity required to distinguish pathological conditions or differences between steroid responsive and nonresponsive patients. Further, fractionation will decrease complexity of the urine proteome while maintaining integrity of the components for more robust identification rates. Although our current methodological study is limited by small sample size, we do utilize a normalization factor derived from clinical information that appears to correct for biological variability within the patient samples. Application of this workflow to a larger sample set should yield novel and important biomarkers to be further validated and studied in depth.

\section{Acknowledgments}

We would like to thank Terri Manning for lab support services and Schweta Tandon for collection of urine samples in the University of Louisville Glomerular Disease Clinic.

\section{Statement of Ethics}

This study was conducted according to the guidelines of the Declaration of Helsinki and approved by the Institutional Review Board (or Ethics Committee) of University of Louisville (protocol 
code: 10.0245; date of approval: February 03, 2017). Clinical samples were obtained from patients followed at the University of Louisville Glomerular Disease Clinic who participate in a voluntary glomerular disease biomarker study. The study is approved by the University of Louisville Institutional Review Board, and all subjects provided consent for research sample collection (IRB\# 01.0536 through 9/16/2022).

\section{Conflict of Interest Statement}

The authors have no disclosures or financial conflicts of interest related to the work presented herein. The authors have no financial disclosures.

\section{Funding Sources}

Funding for this work was partly provided by National Institutes of Health grants DK110077 (J.B.K.), AA024337, AA028436, GM113226 and ES030283 (M.L.M.), and DK126777 (D.W.P.) and a Jewish Heritage Fund for Excellence Award (D.W.P.).

\section{Author Contributions}

Conceptualization: T.D.C., J.B.K., and M.L.M.; methodology: T.D.C., D.W.W., F.W.B., D.W.P., M.T.B., and M.B.; software: T.D.C. and D.W.W.; validation: T.D.C. and M.B.; formal analysis: T.D.C., M.B., D.W.W., and F.W.B.; investigation: T.D.C., D.J.C., D.W.W., M.B., and M.T.B.; resources: J.B.K. and M.L.M.; data curation: T.D.C., M.T.B., and M.B.; writing - original draft preparation: T.D.C.; writing - review and editing: T.D.C., D.W.P., M.L.M., and J.B.K.; visualization: T.D.C., M.T.B., and M.B.; supervision: T.D.C., J.B.K., M.L.M., and D.W.P.; project administration: T.D.C., J.B.K., and M.L.M.; and funding acquisition: J.B.K. and M.L.M. All the authors have read and agreed to the published version of the manuscript.

\section{Data Availability Statement}

Scaffold files from mass spectrometry analysis are readily available in a public database that will be publicly shared at publication: (Cummins, Timothy (2021), "Quantitative Mass Spectrometry Normalization in Urine Biomarker Analysis in Nephrotic Syndrome," Mendeley data, https://data.mendeley.com/datasets/pbxhfvk6r6/draft?a=273f1c77-73b7-4e9e-b0b5-9850f48cbde1).

\section{References}

1 Khanna R. Clinical presentation \& management of glomerular diseases: hematuria, nephritic \& nephrotic syndrome. Mo Med. 2011 Feb;108(1): 33-6.

2 Alsaad KO, Herzenberg AM. Distinguishing diabetic nephropathy from other causes of glomerulosclerosis: an update. J Clin Pathol. 2007 Jan 60(1):18-26

3 Dhaun N, Bellamy CO, Cattran DC, Kluth DC. Utility of renal biopsy in the clinical management of renal disease. Kidney Int. 2014 May; 85(5):1039-48.

4 Schanstra JP, Zürbig P, Alkhalaf A, Argiles A, Bakker SJ, Beige J, et al. Diagnosis and prediction of CKD progression by assessment of urinary peptides. J Am Soc Nephrol. 2015 Aug;26(8): 1999-2010.

5 Critselis E, Vlahou A, Stel VS, Morton RL. Costeffectiveness of screening type 2 diabetes patients for chronic kidney disease progression with the CKD273 urinary peptide classifier as compared to urinary albumin excretion. Nephrol Dial Transplant. 2018 Mar 1;33(3): 441-9.

6 Czech KA, Bennett M, Devarajan P. Distinct metalloproteinase excretion patterns in focal segmental glomerulosclerosis. Pediatr Nephrol. 2011 Dec;26(12):2179-84.

7 Morita M, Uchigata Y, Hanai K, Ogawa Y, Iwamoto Y. Association of urinary type IV collagen with GFR decline in young patients with type 1 diabetes. Am J Kidney Dis. 2011 Dec;58(6):91520.

8 Furumatsu Y, Nagasawa Y, Shoji T, Yamamoto $\mathrm{R}$, Iio K, Matsui I, et al. Urinary type IV collagen in nondiabetic kidney disease. Nephron Clin Pract. 2011;117(2):c160-6.
9 McDowell GS, Gaun A, Steen H. iFASP: combining isobaric mass tagging with filter-aided sample preparation. J Proteome Res. 2013 Aug 2;12(8):3809-12.

10 Li X, Franz T, Atanassov I, Colby T. Step-by-step sample preparation of proteins for mass spectrometric analysis. Methods Mol Biol Clifton NJ. 2021;2261:13-23.

11 Filip S, Vougas K, Zoidakis J, Latosinska A, Mullen W, Spasovski G, et al. Comparison of depletion strategies for the enrichment of low-abundance proteins in urine. PLoS One. 2015;10(7): e0133773.

12 Ganief T, Calder B, Blackburn JM. Protocols for preparation and mass spectrometry analysis of clinical urine samples to identify candidate biomarkers of schistosoma-associated bladder cancer. Methods Mol Biol Clifton NJ. 2021;2292: 143-50.

13 Afkarian M, Bhasin M, Dillon ST, Guerrero MC, Nelson RG, Knowler WC, et al. Optimizing a proteomics platform for urine biomarker discovery. Mol Cell Proteomics. 2010 Oct;9(10): 2195-204.

14 Granger J, Siddiqui J, Copeland S, Remick D. Albumin depletion of human plasma also removes low abundance proteins including the cytokines. Proteomics. 2005 Dec;5(18):4713-8.

15 High AA, Tan H, Pagala VR, Niu M, Cho JH, Wang $\mathrm{X}$, et al. Deep proteome profiling by isobaric labeling, extensive liquid chromatography, mass spectrometry, and software-assisted quantification. J Vis Exp. 2017 Nov 15(129):56474.

16 Pagel O, Kollipara L, Sickmann A. Tandem mass tags for comparative and discovery proteomics. Methods Mol Biol Clifton NJ. 2021;2228:11731.
17 Dayon L, Hainard A, Licker V, Turck N, Kuhn $\mathrm{K}$, Hochstrasser DF, et al. Relative quantification of proteins in human cerebrospinal fluids by MS/MS using 6-plex isobaric tags. Anal Chem. 2008 Apr 15;80(8):2921-31.

18 Huang FK, Zhang G, Lawlor K, Nazarian A, Philip J, Tempst P, et al. Deep coverage of global protein expression and phosphorylation in breast tumor cell lines using TMT 10-plex isobaric labeling. J Proteome Res. 2017 Mar 3;16(3):1121-32.

19 Thompson A, Schäfer J, Kuhn K, Kienle S, Schwarz J, Schmidt G, et al. Tandem mass tags: a novel quantification strategy for comparative analysis of complex protein mixtures by MS/MS. Anal Chem. 2003 Apr 15;75(8):1895-904.

20 Yu Y, Bekele S, Pieper R. Quick 96FASP for high throughput quantitative proteome analysis. J Proteomics. 2017 Aug 23;166:1-7.

21 Froehlich JW, Kostel SA, Cho PS, Briscoe AC, Steen $\mathrm{H}$, Vaezzadeh AR, et al. Urinary proteomics yield pathological insights for ureteropelvic junction obstruction. Mol Cell Proteomics. 2016 Aug;15(8):2607-15.

22 Devarakonda CKV, Shearier ER, Hu C, Grady J, Balsbaugh JL, Makari JH, et al. A novel urinary biomarker protein panel to identify children with ureteropelvic junction obstruction: a pilot study. J Pediatr Urol. 2020 Aug;16(4):466-e9.

23 Kaur G, Poljak A, Ali SA, Zhong L, Raftery MJ, Sachdev P. Extending the depth of human plasma proteome coverage using simple fractionation techniques. J Proteome Res. 2021 Feb 5; 20(2):1261-79.

24 Janciauskiene S, Wrenger S, Immenschuh S, Olejnicka B, Greulich T, Welte T, et al. The multifaceted effects of alpha1-antitrypsin on neutrophil functions. Front Pharmacol. 2018;9:341. 